\title{
THE STRUCTURE OF A LATTICE-ORDERED GROUP AS DETERMINED BY ITS PRIME SUBGROUPS
}

\author{
KEITH R. PIERCE
}

\begin{abstract}
We characterize by structure theorems the classes of all lattice-ordered groups in which (a) every prime subgroup is principal, (b) every proper prime subgroup is principal, and (c) every minimal prime subgroup is principal. These classes are also characterized by the structure of the root system of regular subgroups.
\end{abstract}

In this paper we are concerned with the extent to which the structure of a lattice-ordered group ( $\ell$-group) is determined by the structure of its prime subgroups. In [2], Paul Conrad has shown that each of the convex $\ell$-subgroups of an $\ell$-group $G$ is principal if and only if $G$ is a lex-sum of finitely many $o$-groups and each $o$-group used in the construction of $G$ satisfies the ACC. In Theorem 1 we show that the same class of $\ell$-groups is characterized merely be requiring that all prime subgroups of $G$ be principal. If we relax our requirements slightly by allowing $G$ itself to be nonprincipal, then the class is slightly enlarged to include lex-extensions by certain $o$-groups of $\ell$-groups of the first class (Theorem 3 ). Finally, if only minimal prime subgroups are required to be principal, we get the class of all $\ell$-groups which are lex-extensions of finite lex-sums constructible from principal o-groups (Theorem 2). Furthermore, as in the original case, these classes can be identified by inspecting the lattice of convex $\ell$-subgroups.

First we review here the basic definitions and facts about $\ell$-groups, all of which can be found in [2]. $C$ is a convex $\ell$-subgroup of an $\ell$-group $G$ if $C$ is a subgroup and sublattice of $G$ and is convex in $G$ ( $c \leqq x \leqq d$ and $c, d \in C \Rightarrow x \in C)$. We denote by $\mathscr{C}(G)$ the lattice of all convex $\ell$-subgroups of $G$. A convex $\ell$-subgroup $P$ of $G$ is a prime subgroup of $G$ (in brief, $P$ is prime in $G$ ) if $a \wedge b=0$ implies that one of $a$ and $b$ is in $P$. The set of convex $\ell$-subgroups which include $P$ always forms a chain, and every prime exceeds a minimal prime. $P$ is a regular subgroup of $G$ if it is a convex $\ell$-subgroup which is maximal with respect to not containing some

Received by the editors September 25, 1972 and, in revised form, January 19, 1973. AMS (MOS) subject classifications (1970). Primary 06A55.

Key words and phrases. Lattice-ordered groups, prime subgroups of a lattice-ordered group, lex-sums of ordered groups, lattice-ordered groups with a finite basis. 
element of $G$. Each regular subgroup of $G$ is prime in $G$, and they are distinguishable in $\mathscr{C}(G)$ as the meet-irreducible elements of $\mathscr{C}(G)$. We denote by $\Gamma(G)$ the set of all regular subgroups of $G . \Gamma(G)$ is a root system; that is, the element above a given element of $\Gamma(G)$ form a chain. A root of $\Gamma(G)$ is a maximal chain of $\Gamma(G)$.

$G^{+}$will denote the set of strictly positive elements of $G . G$ is a lexextension of $C$ if $C$ is a prime subgroup of $G$ and $g \in G^{+} \backslash C$ implies that $g>C$. If $G \neq C$ the extension is proper. There is always a smallest convex $\ell$-subgroup $L(G)$ of which $G$ is a lex-extension. $L(G)$ is an $\ell$-ideal of $G$ (normal convex $\ell$-subgroup) and is comparable with every other convex $\ell$-subgroup of $G$. A convex $\ell$-subgroup $D$ of $G$ is a lex-subgroup of $G$ if it is a proper lex-extension of some $C \in \mathscr{C}(G)$, and is a maximal lex-subgroup if it admits no proper lex-extension in $\mathscr{C}(G)$. The set of maximal lexsubgroups is a root system, and any two maximal lex-subgroups are either comparable or disjoint. The polar of $T \subseteq G$ is $T^{\prime}=\{x \in G:|x| \wedge|y|=0$ for all $y \in T\} . T^{\prime}$ is always in $\mathscr{C}(G)$, and for $a \in G$ we will use $a^{\prime}$ to stand for $\{a\}^{\prime}$. An element $a \in G^{+}$is basic if the interval $[0, a]$ is totally ordered. A basis of $G$ is a maximal disjoint collection of positive elements, each of which is basic. $G$ has a basis if and only if every positive element exceeds a basic element. For $a \in G^{+}$, the following are equivalent: $a$ is basic; $a^{\prime}$ is a minimal prime subgroup of $G ; a^{\prime}$ is prime in $G ; a^{\prime \prime}$ is the largest convex $o$-subgroup (i.e., totally ordered convex $\ell$-subgroup) that contains $a$. More generally, a prime $P$ is a minimal prime if and only if every element of $P$ is disjoint from some element outside $P$.

$G$ is a (finite) lex-sum of $o$-groups $\left\{A_{s}: s \in I\right\}$ if there is a finite sequence $L_{1} \subset L_{2} \subset \cdots \subset L_{m}=G$ of $/$-ideals of $G$ such that $L_{k}=\sum A_{k p}\left(p \in I_{k}\right)$, each $I_{k}$ is finite, $I=I_{1}$ and $A_{s}=A_{1 s}$, and if $k>1$ then $A_{k p}$ is either one of the $A_{k-1, q}$ or is a proper lex-extension of the cardinal sum of two or more of the $A_{k-1, a}$ (an $\dot{t}$-group $G$ is the cardinal sum of $f$-groups $B_{k}$, denoted $G=\sum B_{k}$, if $G$ is their direct sum, and the order on $G$ is determined componentwise by the orders on the summands). In a lex-sum, the $A_{k p}$ are precisely all of the maximal lex-subgroups of $G$. The following are equivalent: $G$ is a lex-sum of $n o$-groups; $G$ has $n$, but not $n+1$, disjoint elements; $G$ has a basis of $n$ elements. In this case, the $o$-groups are precisely the $a^{\prime \prime}$, one for each element of the basis.

A convex (-subgroup $C$ of $G$ is said to be generated by $M \subseteq G$ (notation: $C=G(M))$ if $C=\{x \in G:|x| \leqq|y|$ for some $y$ in the subsemigroup of $G$ generated by $M\} . C$ is principal if it is generated by a single element. Thus an $o$-group is principal if it has a largest proper convex subgroup. A lex-subgroup $A$ is principal if and only if $A / L(A)$ is a principal $o$-group.

LEMмA 1. If $C \in \mathscr{C}(G)$ is maximal with respect to being nonprincipal, then $C$ is prime in $G$. 
Proof. If $C$ is not prime then there exist $a, b \in G^{+} / C$ such that $a \wedge b=0$. Thus

$$
\begin{aligned}
C & =G(C \cup\{a \wedge b\})=G(C \cup\{a\}) \cap G(C \cup\{b\}) \\
& =G(g) \cap G(h)=G(g \wedge h),
\end{aligned}
$$

a contradiction.

THEOREM 1. For an $\ell$-group $G$, the following are equivalent:

(1) Each prime subgroup of $G$ is principal.

(2) Each convex $\ell$-subgroup of $G$ is principal.

(3) $\mathscr{C}(G)$ satisfies the $A C C$.

(4) $\Gamma(G)$ has only a finite number of roots and satisfies the $A C C$.

(5) $G$ has a finite basis, and each o-group used in the lex-sum construction of $G$ satisfies the $A C C$.

Proof. The equivalence of (2), (3), (4), and (5) is contained in [2, Theorem 3.10]. It remains to show that $(1) \Rightarrow(2)$. If $G$ has nonprincipal convex $\ell$-subgroups, then an application of Zorn's lemma followed by Lemma 1 yields a nonprincipal prime, completing the proof.

In a lex-sum, we shall frequently encounter subgroups which are cardinal sums of certain of the subgroups $A_{k p}$. We shall call these summands the components of the given subgroup.

Lemma 2. Let $G$ be a lex-sum of the o-groups $\left\{A_{s}: s \in I\right\}$. If $0<a \in A_{s}$ then $a^{\prime}=\sum\left\{A_{k p}: A_{k p} \cap A_{s}=\{0\}\right.$ and either $k=m$ or $A_{k p} \oplus A_{s}$ lies in a component of $\left.L_{k+1}\right\}$ is a minimal prime subgroup of $G$, and every minimal prime is obtained in this fashion. If $a^{\prime}$ and $b^{\prime}$ are different minimal primes, then there is a unique component $A_{l q}$ of $a^{\prime}$ containing $b$, and a unique component $A_{n r}$ of $b^{\prime}$ containing $a$. Furthermore, $G\left(a^{\prime} \cup b^{\prime}\right)$ is the cardinal sum of $A_{l q}, A_{n r}$, and all $A_{k p}$ which are components of both $a^{\prime}$ and $b^{\prime}$.

Proof. The minimal primes in an $\ell$-group with finite basis are of the form $a^{\prime}$, where $a$ is basic. By the proposition on p. 3.32 of [2], $a^{\prime}$ is the cardinal sum of the $A_{\kappa p}$ which are maximal in $a^{\prime}$. These are precisely the components indicated. Since $b$ is basic and lies in $a^{\prime}$, then $b$ lies in a unique component $A_{l a}$ of $a^{\prime}$, and similarly $a$ lies in a unique component $A_{n r}$ of $b^{\prime}$. Clearly the indicated sum $P$ is direct and $P \subseteq G\left(a^{\prime} \cup b^{\prime}\right)$, so it suffices to show that each component $A_{k p}$ of $a^{\prime}$ lies in $P$. If $A_{k p} \subseteq b^{\prime}$ then it can be enlarged to a component $B$ of $b^{\prime}$. If $A_{k p}=B$ then $A_{k p} \subseteq P$. If $A_{k p} \subseteq B$ then $a \in B$, so $B=A_{n r} \subseteq P$. On the other hand, if $A_{k p} \notin b^{\prime}$, then $b \in A_{k p}$, whence $A_{k p}=A_{l q} \subseteq P$.

LeMma 3. If $F$ is an $\ell$-ideal of $G$, then every minimal prime subgroup $Q$ of $G / F$ is of the form $M+F / F$ for some minimal prime subgroup $M$ of $G$. Furthermore, if $M$ is principal, so is $Q$. 
Proof. Write $Q$ as $P / F$ for some prime subgroup $P$ of $G$ containing $F$, and let $M$ be a minimal prime subgroup of $G$ contained in $P$. Then $M+F / F$ is prime, and the minimality of $Q$ forces $Q=M+F / F$. The second statement is straightforward.

THEOREM 2. For an $\ell$-group $G$, the following are equivalent:

(1) Each minimal prime subgroup of $G$ is principal.

(2) $\Gamma(G)$ has a finite number of roots, and if $\Delta$ and $\Lambda$ are two distinct roots of $\Gamma(G)$, then $\Delta \backslash \Lambda$ has a greatest member.

(3) G has a finite basis, and every o-group used in the lex-sum construction of $L(G)$ is principal.

Proof. (1) $\Rightarrow(3)$. We show first that $G$ has a basis. Let $x \in G^{+}$and let $M$ be a minimal prime of $G$ which does not contain $x$. Then $M=G(g)$ for some $g \in G^{+}$. There exists $a \in G^{+} \backslash M$ such that $g \wedge a=0$, whence $M=a^{\prime}$, $a$ is basic, and $x$ exceeds the basic element $a \wedge x$. Since $x$ was arbitrary, $G$ has a basis. Let $\sum A_{s}(s \in I)$ be the basis group of $G$ and let $F=\{x \in G:|x|$ exceeds at most finitely many disjoint elements $\}. F$ is an $\ell$-ideal of $G$ containing $A$, and if $x \in G^{+} \mid F$ then $x$ exceeds some $a_{s} \in A_{s}^{+}$for infinitely many $s$ (see [1, Theorem 6.2]). Suppose $G \neq F$. By Lemma 3, property (1) carries over to the quotient $H=G / F$. In particular, $H=G / F$ has a basis, so we can find $b \in G^{+} \backslash F$ such that $b+F$ is basic in $H$. By Lemma 3 , the minimal prime $(b+F)^{\prime}$ of $H$ is the image of some minimal prime $A_{s}^{\prime}=G\left(g_{s}\right)$ of $G$. Since $(b+F)^{\prime}$ is disjoint from $H(b+F)$, then $G(b) \cap G\left(g_{s}\right)=$ $G\left(b \wedge g_{s}\right) \subseteq F$, which implies that $b \wedge g_{s} \in F$. Now if $t \neq s$ and $b$ exceeds some element of $A_{t}^{+}$, then so does $b \wedge g_{s}$, since $A_{t} \subseteq G\left(g_{s}\right)$. But this occurs for infinitely many $t$, contradicting $b \wedge g_{s}$ being in $F$. Thus $G=F$, but for any $s \in I, A_{s}^{\prime}=G\left(g_{s}\right)$ contains $A_{t}$ for all $s \neq t \in I$, which implies that $g_{s}$ exceeds some element in $A_{t}^{+}$for all $t \neq s$. Since $G=F$, we conclude that $I$ must be finite. Thus $G$ has a finite basis and hence is a lex-sum of the $o$-groups $A_{s}(s \in I)$. It remains to show that if $A_{k p} \neq G$ then it is principal. We may assume that $k$ is the largest index such that $A_{k p}$ is a component of $L_{k}$. If $k=m$ let $A_{k q}$ be any other component of $L_{k}$, otherwise let $A_{k q}$ be another component of $L_{k}$ such that $A_{k p} \oplus A_{k q}$ is bounded in $L_{k+1}$. Let $a$ be a basic element of $G$ in $A_{k q}$. By Lemma $2, A_{k p}$ is a cardinal summand of $a^{\prime}$. Since $a^{\prime}$ is principal, so is $A_{k p}$.

(3) $\Rightarrow(1)$. By Lemma 2 each minimal prime is the cardinal sum of a finite number of principal convex $\ell$-subgroups, and hence is principal.

$(2) \Rightarrow(3)$. By [2, Theorem 3.9], $G$ has a finite basis, and hence is a finite lex-sum of $o$-groups $\left\{A_{s}: s \in I\right\}$. It will suffice to show that if $A_{k p} \neq G$ then it is principal. Let $k$ be the largest index such that $A_{k p}$ is a component of $L_{k}$. If $k=m$ let $A_{k q}$ be another component of $L_{k}$, otherwise let $A_{k q}$ be another component of $L_{k}$ such that $A_{k p} \oplus A_{k q}$ is bounded in $L_{k+1}$. 
Let $M=a^{\prime}$ and $N=b^{\prime}$ where $a \in A_{k p}$ and $b \in A_{k q}$ are basic elements of $G$. By hypothesis, there is a regular subgroup $P$ which includes $M$ and is the largest such subgroup which does not include $N$. It follows that $M \subseteq P \subset$ $G(M \cup N)$. We refer to the cardinal decomposition of $G(M \cup N)$ given in Lemma 2 , and recall that, in a cardinal sum, a convex $\ell$-subgroup is the cardinal sum of its intersection with each of the summands. Since $A_{k p}$ is the only component of $G(M \cup N)$ which is not contained in $M$, it follows that $P \cap A_{k p}$ is the unique largest convex $\ell$-subgroup of $A_{k p}$. Since we must have $L\left(A_{k p}\right) \subseteq P \cap A_{k p} \subset A_{k p}$, it follows that $A_{k p}$ is principal.

(3) $\Rightarrow(2)$. Let $M=a^{\prime}$ and $N=b^{\prime}$ be unique minimal prime subgroups of $G$ which are included respectively in each element of $\Delta$ and each element of $\Lambda$. By Lemma 2 let $A_{k p}$ be the unique component of $G(M \cup N)$ which contains $a$. By hypothesis $A_{k p}$ has a largest proper convex $\ell$-subgroup $B$. If $A_{k p}$ is replaced by $B$ in the cardinal decomposition of $G(M \cup N)$, we arrive at the desired greatest member of $\Delta \backslash \Lambda$.

LEMMA 4. For an o-group $H$ the following are equivalent:

(1) Every proper convex subgroup of $H$ is principal.

(2) Every proper convex subgroup of $H$ has the $A C C$.

(3) The chain of convex subgroups of $H$ is a finite or denumerable lexicographic union of inversely well-ordered sets.

Proof. $\quad(1) \Rightarrow(2)$. Immediate.

$(2) \Rightarrow(3)$. Let $H_{1}$ be a proper convex subgroup of $H$. By finite recursion form a finite or denumerable chain of convex subgroups $H_{1} \subset H_{2} \subset \cdots$ as follows: if $H_{n}$ has been constructed and $H_{n}=H$, stop. If $H$ covers $H_{n}$ let $H_{n+1}=H$. Otherwise choose $H_{n} \subset H_{n+1} \subset H$. In any case we have a chain of convex subgroups each of which has the ACC and whose union must be $H$. If for each $n$ we let $\mathscr{C}_{n+1}$ be the set of all convex subgroups $C$ of $H$ such that $H_{n} \subseteq C \subset H_{n+1}$ then each $\mathscr{C}_{n}$ is inversely well-ordered, and the union of all the $\mathscr{C}_{n}$ is the set of all proper convex subgroups of $H$.

$(3) \Rightarrow(1)$. Since the lexicographic union of finitely many inversely well-ordered sets is an inversely well-ordered set, each proper convex t-subgroup of $H$ will in fact have the ACC and hence be principal.

To simplify the statement of the next theorem we introduce some notation. If $\Gamma$ is a root system then there is a largest subset $\Lambda$ of $\Gamma$ with the property that $\Lambda$ is a chain and $\alpha<\Lambda$ whenever $\alpha \notin \Lambda$. We denote by $L(\Gamma)$ the set $\Gamma \backslash \Lambda$ and make the observation that if $\Gamma=\Gamma(G)$ then $L(\Gamma)=\{P \in \Gamma: P \subseteq L(G)\}$. Also, each root $\Delta$ of $\Gamma$ is the disjoint union of $\Lambda$ with the root $\Delta \cap L(\Gamma)$ of $\Gamma$.

THEOREM 3. For an l-group $G$ the following are equivalent:

(1) Every proper prime subgroup of $G$ is principal. 
(2) $\Gamma(G)$ has finitely many roots, $L(\Gamma(G))$ has the $A C C$, and the chain $\Gamma(G) \backslash L(\Gamma(G))$ either has the ACC or is a denumerable lexicographic union of chains having the $A C C$.

(3) $G$ has a finite basis, each o-group used in the lex-sum construction of $L(G)$ has the $A C C$, and every proper convex subgroup of the o-group $G / L(G)$ is principal.

Proof. $\quad(1) \Rightarrow(3)$. By Theorem 2, $G$ has a finite basis and each $o$-group used in the lex-sum construction of $L(G)$ is principal. But then $L(G)$ is principal, being a finite sum of principal lex-subgroups. Thus every prime subgroup of $L(G)$ is principal, so by Theorem 1 each $o$-group used in the lex-sum construction of $L(G)$ has the ACC. Finally, each proper convex subgroup of $G \backslash L(G)$ is the image of a proper prime subgroup of $G$, and hence is principal.

$(3) \Rightarrow(2)$. Since $G$ has a finite basis, $\Gamma(G)$ has finitely many roots. By Theorem $1 L(\Gamma(G))$ has the ACC. The last statement follows from Lemma 4.

$(2) \Rightarrow(1)$. By Theorem 1 every prime subgroup of $L(G)$ is principal, and by Lemma 4, every proper prime subgroup of $G$ which contains $L(G)$ is principal. These are all of the proper primes of $G$.

\section{BIBLIOGRAPHY}

1. P. Conrad, Some structure theorems for lattice-ordered groups, Trans. Amer. Math. Soc. 99 (1961), 212-240. MR 22 \#12143.

2. - Lattice-ordered groups, Tulane University Lecture Notes, 1970.

Department of Mathematics, University of Missouri, Columbia, Missouri 65201 